\title{
HIGH POWER RF CONDITIONING OF THE LEDA RFQ*
}

\author{
L. M. YOUNG, D. E. REES, L. J. RYBARCYK, and K. A. CUMMINGS, \\ Los Alamos National Laboratory, Los Alamos, NM, 87545
}

\begin{abstract}
We are preparing the radio frequency quadrupole (RFQ) for the Low Energy Demonstration Accelerator (LEDA) [1] to accelerate beam. The LEDA RFQ accelerates a 100-mA CW proton beam from $75 \mathrm{keV}$ to $6.7 \mathrm{MeV}$. We will report our experience with high-power RF conditioning the RFQ, first with one klystron and then with two klystrons. The RFQ will dissipate 1.2 megawatts of RF power at design fields. This $350-\mathrm{MHz}$ CW RFQ [2] has peak fields on the vane tips of $33 \mathrm{MV} / \mathrm{m}$. The average power dissipation is $13 \mathrm{watts} / \mathrm{cm}^{2}$ on the outer walls of the RFQ near the high energy end. The power from each klystron is split 4 ways to lower the stress on the RF windows. Each klystron can produce 1.3 megawatts of RF power.
\end{abstract}

\section{INTRODUCTION}

The LEDA RFQ consists of four 2-meter-long RFQs resonantly coupled together to form an 8-meter-long structure. These sections are labeled A, B, C, and D starting from the low-energy end. RF drive ports are located in the $\mathrm{B}, \mathrm{C}$ and $\mathrm{D}$ sections. The RF power is coupled into the RFQ through half-height WR2300 waveguide and a section of tapered ridge-loaded waveguide to a coupling iris. The tapered waveguide has the dimensions of the half-height WR2300 waveguide at one end and tapers to only 7 inches wide at the iris to the RFQ as shown in Figure 1. The gap between the ridges of the ridge-loaded waveguide slowly increases as the cross section increases in size toward the half-height WR2300 waveguide.

RF conditioning of the LEDA RFQ started on November 19, 1998. At that time one klystron was connected through a network of waveguide splitters to the four window-waveguide assemblies that feed RF power to section $\mathrm{B}$ of the RFQ. The four RF drive ports in section $\mathrm{C}$ were filled with water-cooled plugs. The four RF drive ports in section $\mathrm{D}$ had the lower part of the waveguide taper with the coupling iris installed. A shorting plate affixed to each tapered waveguide positioned the short at $\lambda_{\mathrm{g}} / 4$ from the iris where $\lambda_{\mathrm{g}}$ is the wavelength of the 350 $\mathrm{MHz}$ RF in the waveguide. This arrangement makes each section of waveguide anti-resonant, which results in no net power flow into these waveguides.

\section{INITIAL CONDITIONING}

After the initial demonstration, High Power RF (HPRF) conditioning began in earnest about 9 AM on 11/20/98. For the first hour of conditioning, the RF power level was set at 3 to $5 \mathrm{~kW}$. Multipacting occurred in the vacuum waveguides, most likely near the iris where the gap between the tapered waveguides is slightly larger than the 1/16-inch gap at the iris. This gap increases slowly with distance from the iris. The evidence for this multipacting is the low power level at which the multipacting occurred and the increase in the pressure in the RFQ and the vacuum waveguides. This multipacting disappeared after about an hour and the RF power level was relatively quickly increased to $10 \mathrm{~kW}$ as the pressure in the RFQ and waveguides permitted. At the $10 \mathrm{~kW}$ power level, the first radiation survey was performed, and as expected, nothing above background was detected outside the accelerator tunnel exclusion area. Radiation surveys were performed at $10,30,50$, and $70 \mathrm{~kW}$ power levels; nothing above background was detected.

The pressure in the vacuum waveguides paced the rate at which we could increase the RF power. A vacuum interlock system used the pressure readings from ion gauges on the RFQ vacuum manifold and on each of the vacuum waveguides to turn off the RF power whenever one of them indicated poor vacuum. The vacuum interlock

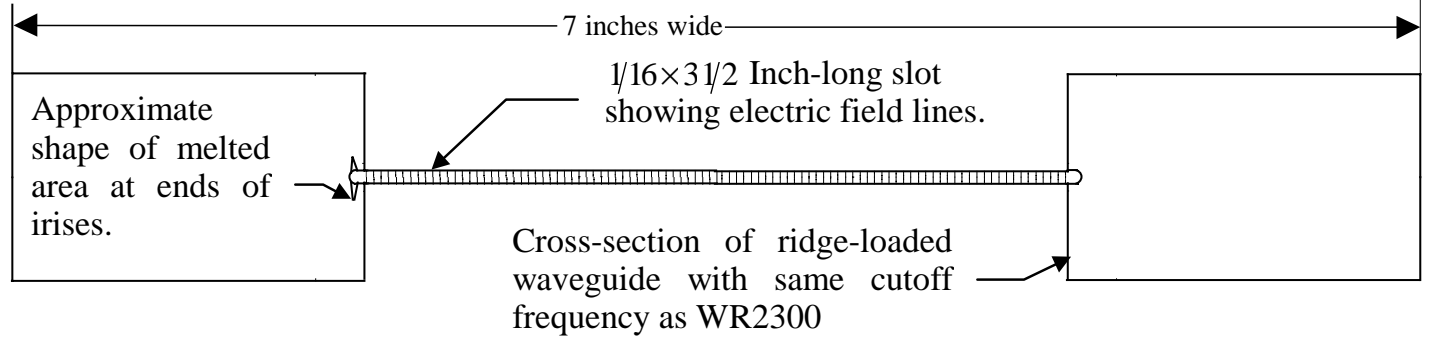

Figure 1 Cross section of ridge-loaded waveguide and dimensions of iris. The iris is a slot 3.5 inches long with a $\sim 1 / 16$-inch diameter hole at each end.

*Work supported by the US Department of Energy. 
thresholds on the RFQ and waveguide were set at $2 \times 10^{-6}$ and $5 \times 10^{-6}$ Torr, respectively. On the waveguides this interlock protects the windows from damage that could result from RF power being present under poor vacuum conditions.

The "Blanking Box" (formerly used to protect the CRITS RFQ [3]) was used during the conditioning process to protect the RFQ from internal arcs. This circuit interrupts the drive to the klystrons for $200 \mu$ s whenever the reflected power exceeds a specified level.

By midnight November $23^{\text {rd }}$, a forward power level of $100 \mathrm{~kW}$ had been reached with waveguide vacuum on quadrant 2 at $5 \times 10^{-6}$ Torr. The pressure in this vacuum waveguide had limited the rate of progress all evening. Various processing schemes were tried, e.g. no amplitude modulation (AM), processing with up to $100 \%$ AM (sine wave) and processing with an AM consisting of $100 \mathrm{~Hz}$, 1-2 ms long pulses on top of an 85-90 kW CW background. None of these methods seemed to help increase the sustainable RF power level. This stage of the conditioning process proceeded rather slowly.

\section{MULTIPACTING}

We believe that multipacting in the tapered section of the waveguide caused the slow progress in conditioning the RFQ. At low RF power levels the multipacting occurs close to the iris where the gap is slightly greater than $1 / 16^{\text {th }}$ inch. As the RF power increases, the position where multipacting can occur moves up the taper toward the WR2300 waveguide. At the halfway point in the tapered section the gap is $2.9 \mathrm{~cm}$ and simple multipacting theory indicates that multipacting can occur with the RF power equal to $68 \mathrm{~kW}$ with VSWR $=1$. This theory indicates that this power level is about the limit for the simple half cycle multipacting. However, it will occur at higher power levels when the VSWR>1 and the waveguide is over coupled to the RFQ.

Our current plan to mitigate the multipacting problem reduces the number of RF waveguide feeds by a factor of two. We will use one klystron on section B and only feed quadrants 3 and 4 . On section $D$ we will use two klystrons, each feeding two quadrants. Our original plan used one klystron per section to drive sections B, C, and $\mathrm{D}$ with each klystron driving 4 quadrants. Currently with two klystrons attached to the RFQ we must drive the RFQ with at least $800 \mathrm{~kW}$ to be above the power level where multipacting is a problem. If we stayed with our original plan, the multipacting would occur at up to $1.2 \mathrm{MW}$ with 3 klystrons. This power is our design power level without beam. By halving the number of waveguide feeds, we will be above the multipacting level at $600 \mathrm{~kW}$.

\section{HIGH POWER CONDITIONING}

Conditioning with high power RF pulses began on November $24^{\text {th }}$. By setting the peak RF power to $>400$ $\mathrm{kW}$ and limiting the pulse length to $67 \mu$ s we were able to break through the multipacting in the waveguides. Initially, the pulse length was limited to $67 \mu$ s when the Blanking Box, which was tripping on the fast RF turn-on, was disabled. Later we modified the Blanking Box to ignore these turn-on transients. We were able to increase the power level to $600 \mathrm{~kW}$ with $67-\mu \mathrm{s}$ pulses and $33 \mu \mathrm{s}$ between pulses on this day.

The next day the peak power was raised to $958 \mathrm{~kW}$ at $12 \%$ duty factor. On both of these two days the conditioning was paced by vacuum in the RFQ. At the high peak power levels the waveguides were not multipacting and the vacuum remained good in the waveguides.

\section{WINDOWS INSTALLED ON D-SECTION.}

The four D-section windows were installed during the first week in December. Because we were not ready to connect a second klystron to the RFQ and the length of the vacuum waveguide and window was effectively $\lambda_{\mathrm{g}}$, we installed a piece of $\lambda_{\mathrm{g}} / 4$ waveguide and a short to make each waveguide coupled to section D anti-resonant again. The following week was spent on RF conditioning the vacuum waveguide on section $\mathrm{D}$. Even though there was no klystron connected to the D-section waveguide, the RFQ transmits RF power into the waveguide. This power reflects off the short creating a standing wave in the waveguide. Because of the standing wave multipacting can occur in the tapered waveguide section. By the end of the second week in December the RFQ and waveguides could be operated at power levels up to $100 \mathrm{~kW} \mathrm{CW}$ from the klystron driving section B. On December $16^{\text {th }}$, the RF power was then increased above the multipacting band to operating levels of $800 \mathrm{~kW} \mathrm{CW}$ and up to $900 \mathrm{~kW}$ peak with a pulsing format.

\section{TWO KLYSTRON OPERATION}

A second klystron was connected to the D-section waveguide network December $17^{\text {th }}$. Continued operation required phase control of the second klystron with respect to the first klystron. By December $24^{\text {th }}$, the RFQ was conditioned to about 1.08 MW average power in a pulsed format with about 1.1-1.2 MW peak.

\section{IRIS MELTING}

The conditioning process resumed on January $5^{\text {th }}$ with further operation at $1 \mathrm{MW} \mathrm{CW}$ power levels in the RFQ. On January $7^{\text {th }}$ a valve was discovered in the closed position preventing cooling from flowing in the vane-tip cooling channels in section C. Although no damage to the RFQ occurred as a result of this lack of cooling, the fields at the D1 waveguide irises were about $10 \%$ higher than normal. Subsequently, when the RFQ was conditioned with the proper level of cooling in section C, 1.2 MW of net RF power in the RFQ was achieved.

However, the reflected power on the D-section waveguide network was now about $10 \%$ instead of the $2 \%$ observed on December $24^{\text {th }}$. Also, the frequency of the RFQ was $80 \mathrm{kHz}$ lower than previously measured. In the process of looking for the cause of the high-reflected power we removed the window on quadrant 3 of section D and discovered that some melting had occurred at both ends of the iris as shown on Figure 1. Further 
investigation revealed that waveguide iris in quadrant 2 of section D also showed evidence of melting. None of the B-section irises or quadrant 1 and 4 irises of section D displayed any evidence of melting. The RF field strength in section B is $10 \%$ lower than section D. Also, the field strength in quadrants 2 and 3 were slightly higher than in quadrant 1 and 4 of section D.

A MAFIA calculation on a simplified model of the iris showed the current at the ends of the iris slot was enhanced a factor of 10 over the RFQ wall current. The copper iris plate was only $1 / 16$ inch thick at this point. This localized heating coupled with the increase in surface resistance at elevated temperatures was enough to melt the ends of the iris slots when the RFQ was operated CW at close to the design fields. The model results also showed that by increasing the thickness of the iris plate to $3 / 8$ inch, the size of the hole at the end of the slot doubles for the same coupling. This change will reduce the enhancement of the wall currents from a factor of 10 to only 2.5. We will replace the present $1 / 16$-inch-thick iris plates with iris plates that are 3/8-inch thick.

\section{RETUNING}

The RFQ was retuned in January because the drop in frequency of $80 \mathrm{kHz}$ (caused by the iris melting) was believed to have resulted in a change in the field distribution. A bead perturbation measurement confirmed that the field distribution had changed a small amount. By adjusting the length of only 21 of the 128 tuners we restored the fields to within $1 \%$ of the original tune [4] performed before the waveguide coupling irises were installed.

\section{CONDITIONING AND BEAM}

During the final conditioning from January 27 to March 15, 1999 we restricted the RF power dissipated in the RFQ to 1.2 MW or less. This restriction required conditioning the RFQ with a pulsed format whenever we raised the RF fields above the design value. During this period we integrated the low-level RF (LLRF), the HPRF, and the water cooling system to reliably maintain the RF fields in the RFQ. The LLRF system sends a frequency error signal to the RFQ's water cooling system. This resonance control cooling system (RCCS) adjusts the temperature of the water cooling the outer walls of the RFQ to maintain the resonant frequency of the RFQ at $350 \mathrm{MHz}$. The cooling system also supplies $50^{\circ} \mathrm{F}$ water to cooling channels near the vane tips.

By March $15^{\text {th }}$ the RFQ would run for long periods of time with $1.1 \mathrm{MW}$ average power. On March $16^{\text {th, }}$ a 6-mA beam pulsed at $5 \mathrm{~Hz}$ and $300 \mu$ s duration was injected into the RFQ for the first time. We pulsed the RF in the RFQ to $\sim 1.2 \mathrm{MW}$ at $500 \mathrm{~Hz}$ and $90 \%$ duty factor. The RF pulses were 1.8 milliseconds long. Without steering the beam and with the initial settings of the focusing solenoids in the injector beam line, $4 \mathrm{~mA}$ of beam at $6.7 \mathrm{MeV}$ was obtained that first day [5]. By March $19^{\text {th }}$ we had increased the injected current to $10 \mathrm{~mA}$ with $\sim 100 \%$ transmission.

\section{ACKNOWLEDGEMENTS}

We would like to thank Frank Krawczyk for performing the MAFIA calculations to help resolve the iris-melting problem. In addition to the large number of people at LANL, we would also like to acknowledge other institutions participating in the LEDA project including LLNL and TECH-SOURCE (vacuum system), and AlliedSignal (RCCS).

\section{REFERENCES}

[1] H. V. Smith, Jr. and J. D. Schneider, Status Update on the Low-Energy Demonstration Accelerator (LEDA)," Proc. 1998 LINAC Conf. [Chicago, 24-28 August 1998] (in press).

[2] D. Schrage et al., "CW RFQ Fabrication and Engineering," Proc. 1998 LINAC Conf. [Chicago, 24-28 August 1998] (in press).

[3] G. M. Arbique et al., "Beam Parameter Measurements on the CW RFQ-1 Accelerator," Proc. 1992 LINAC Conf. (AECL-10728, November, 1992) 55-57.

[4] L. M. Young and L. Rybarcyk, "Tuning the LEDA RFQ 6.7 MeV Accelerator," Proc. 1998 LINAC Conf. [Chicago, 24-28 August 1998] (in press).

[5] K. F. Johnson et al., "Commissioning of the LowEnergy Demonstration Accelerator (LEDA) RadioFrequency Quadrupole (RFQ)," this Conf. 